Check for updates

Cite this: RSC Adv., 2019, 9, 15099

\title{
Repair of dentine-related lesions without a drill or injection
}

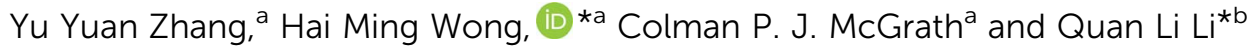 \\ For covering the shortages of traditional treatments, a novel and non-invasive system was developed with \\ the simple adaption of nature's own repair process, while an extrinsic electric field was introduced to \\ improve its remineralization kinetics. In an in vivo study, acid-etched rabbit dentine was used to evaluate \\ the remineralization efficacy and safety of the system. The exposed dentine tubules were fully occluded \\ after 5 hours $/ 1.0 \mathrm{~mA}$ and 8 hours $/ 0.5 \mathrm{~mA}$ of remineralization. After 5 hours of remineralization $(1.0 \mathrm{~mA})$, \\ the micro-hardness of the demineralized dentine was fully recovered, equal to native rabbit dentine. \\ Haematoxylin-eosin staining demonstrated no obvious inflammatory reaction. This study provides \\ a feasible solution to realize rapid repair of dentine.
}

Received 12th April 2019

Accepted 6th May 2019

DOI: 10.1039/c9ra02759a

rsc.li/rsc-advances

Lots of efforts have thus been directed toward finding

\section{Introduction}

Teeth are the most highly mineralized organs in the human body. Their outmost layer is enamel, which is more than $95 \%$ inorganic crystalline hydroxyapatite, protecting the inner dentine and dental pulp from harm and stimuli. ${ }^{1,2}$ Dentine is a collagenous mineralized tissue that contains approximately $70 \%$ carbonated apatite, $20 \%$ organic matrix, and $10 \%$ water by weight. ${ }^{3}$ Almost all human beings have dental hard tissue lesions, with various levels of severity, due to congenital defects, caries, erosion, attrition, trauma, etc. Due to the fear of the dental drill and anesthetic injections, there are quite a few people who do not attend dental appointments. It is not surprising that the dental check-up ranks as the top of a list of dreaded activities, even higher than public speaking. ${ }^{4,5}$ Thus, most dental hard tissue lesions are not restored/repaired at the initial stage and gradually become larger and symptomatic. Additionally, the traditional treatment for dental hard tissue lesions is an invasive therapy, including preparing a cavity on sound dental hard tissue and restoring it with conventional materials ${ }^{6}$ such as mercury amalgam, ${ }^{7}$ composite resin ${ }^{8}$ and glass ionomer cement. ${ }^{9}$ However, due to the differences in properties between dental hard tissue and artificial materials, the long-term performance of the restored tooth is far from our expectation. Restorative failures such as marginal degradation/ leakage, recurrent/secondary caries, gingival/pulpal irritation, and corrosion, fracture, discoloration and dislodgment of restorations are commonly encountered at the dental clinic.

${ }^{a}$ Faculty of Dentistry, The University of Hong Kong, The Prince Philip Dental Hospital, 34 Hospital Road, Hong Kong. E-mail: wonghmg@hku.hk; Fax: +852 25593803; Tel: +852 28590261

${ }^{b}$ Department of Prosthodontic, College and Hospital of Stomatology, Anhui Medical University, No. 69, Meishan Road, Hefei, China. E-mail: ql-li@126.com; Fax: +86 o551 5111538; Tel: +8605515118677 alternative treatment strategies, and one of those is biomimetic mineralization. The biomineralization strategy aims at introducing calcium and phosphate ions to induce recrystallization in lesions and to replace demineralized tissue. However, the rate of crystal growth is very slow, which limits its clinical applicability. Our team has been dedicated to the development of minimally invasive treatments for dental hard tissue lesions through biomimetic mineralization. We have successfully established an electrophoresis-aided mineralization system for regenerating enamel-like structure on enamel lesions. ${ }^{10}$ It was identified that ion migration is excited, greatly improving the mineralization efficiency, under the influence of an extrinsic electric field. However, due to the complex structure and higher organic composition of dentine, ${ }^{11}$ its remineralization is totally different from that of enamel; namely, dentine biomineralization is much more difficult to achieve in comparison to enamel biomineralization. ${ }^{12-16}$ The goals of the remineralization of enamel lesions and dentine lesions are also different. The goal of remineralization of an enamel lesion is to fill the lesion with minerals, ideally penetrating the subsurface area of the lesion so as to obtain stronger resistance against subsequent caries attack than that of the original enamel tissue. The goals of remineralization of dentine include (i) remineralizing the demineralized dentine tissue, (ii) occluding open dentinal tubules by the remineralization process, and (iii) formation of a layer of enamel-like tissue on the remineralized dentine surface to protect the dentine-pulp complex from caries, erosion and attrition. ${ }^{17}$

Root caries has a high incidence among elderly people. ${ }^{18,19}$ With a quickly growing elderly population, the difficulty and cost of treatment for root caries have become a heavy burden in modern society. Dentine is the main affected tissue in root caries, and also in dental hypersensitivity (DH). DH is 
a common oral symptom presented with a rapid sharp pain and a short duration. The reported prevalence of $\mathrm{DH}$ is as high as 40-60\% among 18-65 year old patients. ${ }^{20,21}$ The pain caused by DH can severely impair oral health-related quality of life. ${ }^{22}$ The effect of current treatment for root caries and DH is temporary and limited. Therefore, the investigation of dentine remineralization is highly demanded, and it is also more challenging compared with that of enamel remineralization. To tackle those challenges, we established the rabbit model of exposed/ demineralized dentine for in vivo evaluation of the electrophoresis-aided mineralization system. Since the influence of electrophoresis on pulpal tissue for dentine remineralization was more severe than that for enamel remineralization, an in vivo pulpal tissue response was investigated to measure the safety parameters for future clinical applications. This study provides a novel and stress-free dental treatment option for dentine lesions without any invasive operations.

\section{Materials and methods}

Twenty-eight male New Zealand rabbits with similar teeth size (age: 90-120 days; body weight: 2.065-2.309 kg; the Department of Animal Experimental Centre of Anhui Medical University (certificate number SYXK (Wan) 2013-004)) were used in this study. The Anhui Medical University Experimental Animal Ethics Committee approved all the animal-related experimental procedures, and all these procedures were carried out in accordance with the National Institutes of Health guide for the care and use of laboratory animals (NIH Publications no. 8023, revised 1978).

\subsection{The preparation of the casein phosphopeptide-calcium phosphate (CPP-ACP) suspension}

The CPP-ACP suspension was prepared by mixing $1 \mathrm{~g}$ of CPPACP paste (Tooth Mousse, GC Corp., Tokyo, Japan) with $1 \mathrm{~mL}$ of remineralization solution $\left(2.58 \mathrm{mM} \mathrm{CaCl}_{2} \cdot 2 \mathrm{H}_{2} \mathrm{O}, 1.55 \mathrm{mM}\right.$
$\mathrm{KH}_{2} \mathrm{PO}_{4}, \quad 50 \quad \mathrm{mM}$ trihydroxymethylaminomethane(tris)hydrochloric, $180 \mathrm{mM} \mathrm{NaCl}$ and $1 \mathrm{mg} \mathrm{L}^{-1} \mathrm{NaF}$ ) at $\mathrm{pH} 7.6 .^{23}$

\subsection{The design of a personalized mould for the loading of the CPP-ACP suspension}

A personalized mould was formed and fixed on the maxillary incisors to load the CPP-ACP suspension (Fig. 1C and D). First, a plaster model of a rabbit's maxillary incisors was made by using alginate to make an impression of the incisors. Then, the second impression on the plaster model was created by using the heavy body of hydrophilic addition-type silicone-rubber impression materials (3M ESPE, Seefeld, Bayern, Germany). Lastly, the personalized mould with an opening cavity on the labial surface was formed by removing the labial siliconerubber of the second impression.

\subsection{The preparation of an acid-etched dentine surface on a rabbit's maxillary incisors}

The labial surface of the maxillary incisor was prepared by a high-speed turbine hand piece to remove its enamel and expose the dentine (Fig. 1A and B). Then, the labial surface of the prepared incisor was etched with $37 \%$ phosphoric acid gel (Gluma Etch 35 Gel, Heraeus Kulzer GmbH, Germany) for 15 second and the surface was rinsed with a large volume of deionized water. After these two steps, the acid-etched labial dentine surface of the maxillary incisor was formed.

\subsection{The assembly of the remineralization device on the rabbit incisors}

Four test groups, containing 28 rabbits with acid-etched maxillary incisor dentine in all, were used in this study. Those in experimental group A were remineralized with the CPP-ACP suspension aided with $1.0 \mathrm{~mA}(n=9)$. Those in experimental group B were remineralized with the CPP-ACP suspension aided with $0.5 \mathrm{~mA}(n=9)$. Rabbits in the control group were
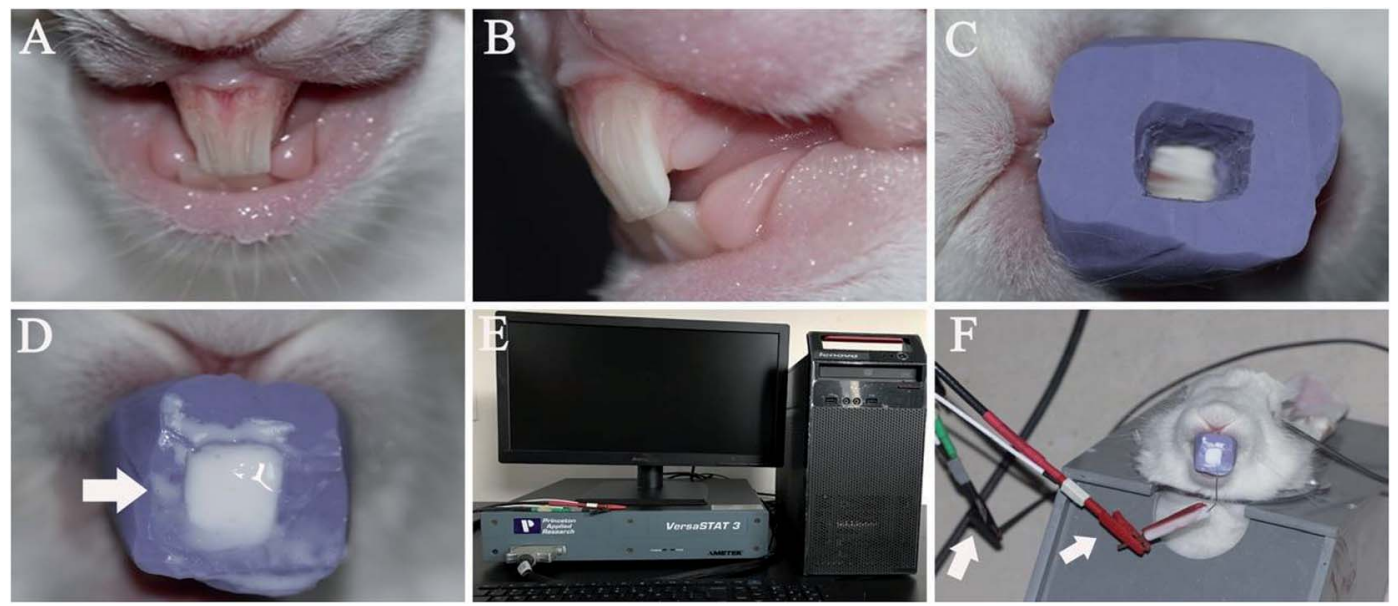

Fig. 1 Remineralization of acid-etched dentine on rabbit's maxillary incisors. (A) The frontal view of the prepared incisors; (B) the lateral view of the prepared incisors; (C) the personalized mould with an opening cavity on the labial surface fixed on the prepared incisors; (D) the personalized mould loaded with the CPP-ACP suspension (arrow 1, CPP-ACP suspension); (E) Electrochemistry workstation (VersaSTAT3, AMETEK Inc., America); (F) the assembling of the electrophoresis-aided CPP-ACP remineralization system (arrow 1, anode; arrow 2, cathode). 

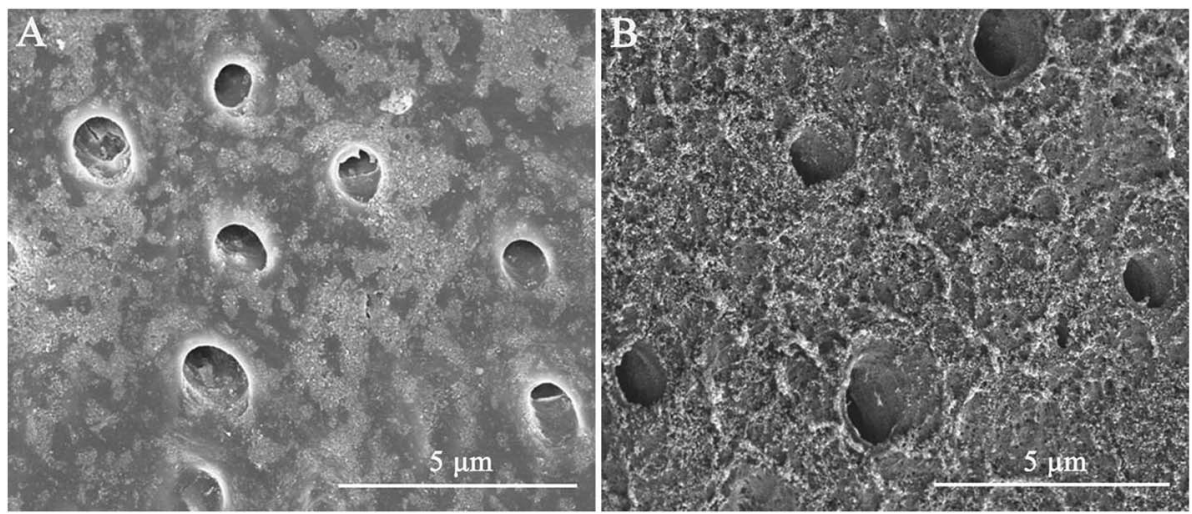

Fig. 2 SEM micrographs of acid-etched dentine and remineralized dentine in the control group. (A) The SEM micrograph of acid-etched dentine on a rabbit incisor; (B) the SEM micrograph of remineralized dentine in the control group after 8 hours remineralization.

remineralized with the CPP-ACP suspension $(n=9)$, while the rabbit in the blank control group received no treatment $(n=1)$.

The acid-etched dentine of the 18 rabbits in group A and B was remineralized under galvanostatic conditions in a twoelectrode system. The skin of the rabbits' heads was exposed by depilatory wiping off of their fur and connecting the skin of the head to the anodes. The cathode was inserted into the personalized mould loaded with CPP-ACP suspension, which was then fixed in the acid-etched dentine of the maxillary incisors (Fig. 1F). A constant electric current of $1.0 \mathrm{~mA}$ or $0.5 \mathrm{~mA}$ was controlled through an electrochemistry workstation (VersaSTAT3, AMETEK Inc., America) (Fig. 1E). The acid-etched dentine of the nine rabbits in the control group was remineralized using the CPP-ACP suspension only. The rabbit in the blank control group was used to detect the demineralized dentine surface.
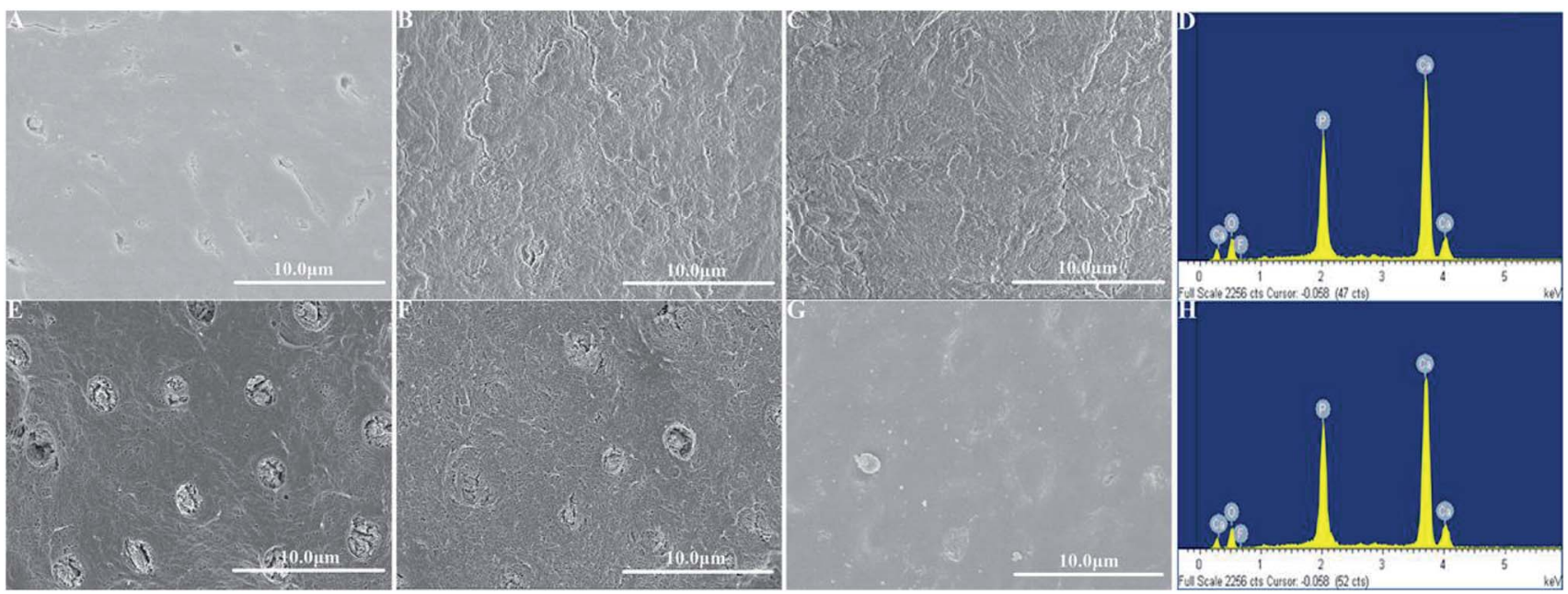

Fig. 3 SEM micrographs of remineralized dentine in the experimental groups. (A) The SEM micrograph of remineralized dentine in group A after 3 hours remineralization; (B) the SEM micrograph of remineralized dentine in group A after 5 hours remineralization; (C) the SEM micrograph of remineralized dentine in group A after 8 hours remineralization; (D) EDS spectra of the remineralized dentine in group $A$ after 8 hours remineralization; (E) the SEM micrograph of remineralized dentine in group B after 3 hours remineralization; (F) the SEM micrograph of remineralized dentine in group $B$ after 5 hours remineralization; $(G)$ the SEM micrograph of remineralized dentine in group $B$ after 8 hours remineralization; $(H)$ EDS spectra of the remineralized dentine in group $B$ after 8 hours remineralization. 

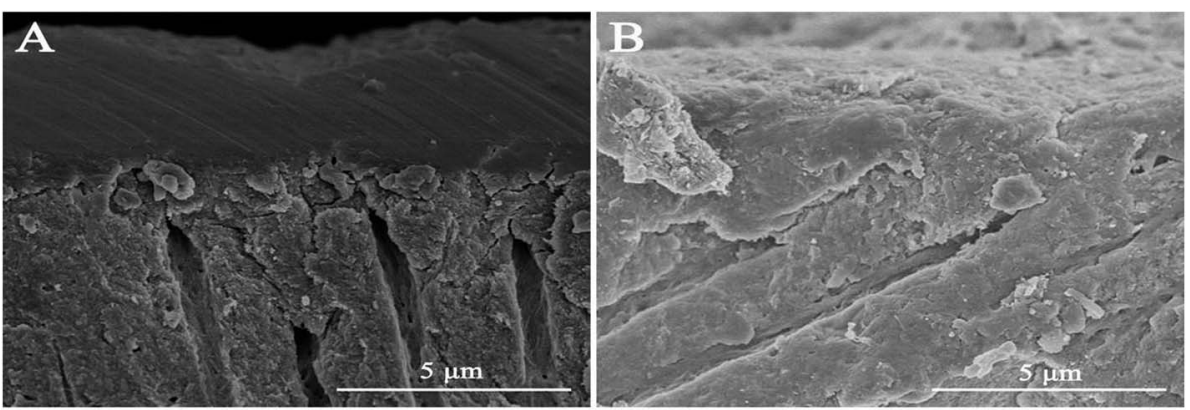

Fig. 4 The transversal micrographs of remineralized dentine in the experimental groups. (A) Transversal micrograph of remineralized dentine in group A after 5 hours mineralization; (B) transversal micrograph of remineralized dentine in group B after 8 hours mineralization.

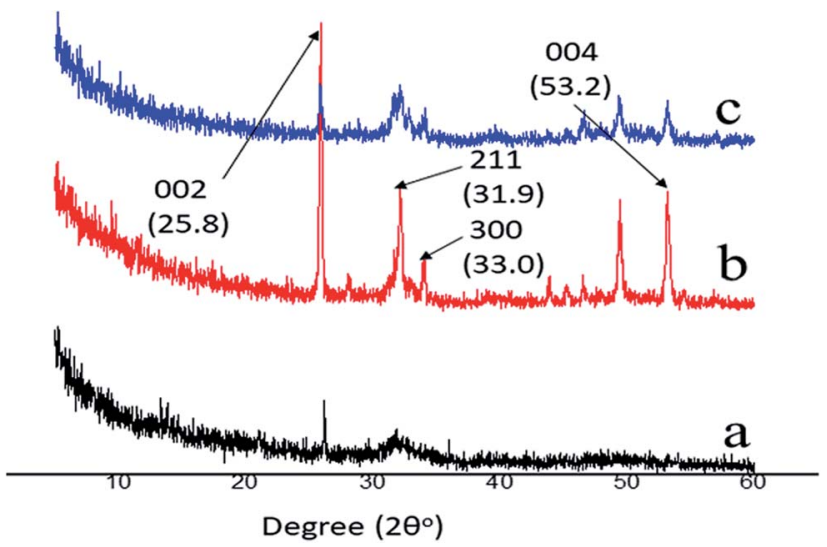

Fig. 5 XRD patterns of native, acid-etched and remineralized dentine (a) represents the native rabbit dentine; (b) represents the remineralized dentine in group B after 5 hours remineralization; (c) represents the remineralized dentine in group $A$ after 5 hours remineralization.

200, USA), respectively. The structure of new crystals on remineralized dentine was identified using X-ray diffraction (XRD) (X'Pert Pro, Philips Almelo, Netherlands). Before the SEM, EDS and XRD evaluation, we dehydrated all the samples with gradual ethanol and dried them in a critical evaporator. The mechanical properties of the rabbits' native dentine, acidetched dentine and remineralized dentine were evaluated using a micro-hardness tester (Leica DC 300, Leitz, Germany). Before the testing, the Knoop tip was calibrated with a standard calibration reference block. Six test points with $30 \mathrm{~mm}$ spacing on the surface of the sample were evaluated. The data were recorded and analyzed with statistic software (SPSS Statistic 24;
IBM). Differences were considered significant at $p<0.05$. Data were expressed as mean \pm standard deviation.

\subsection{The histological examination}

One rabbit was randomly chosen from each subgroup in group A, group B and the control group. Nine rabbits with eighteen maxillary incisors in total were used for a histological evaluation. Extracted maxillary incisors were fixed and decalcified in 15\% EDTA for 3 weeks. After decalcification, all samples were dehydrated, cleaned and then embedded in paraffin. In each sample, serial sections $5 \mu \mathrm{m}$ thick were made in the buccolingual direction, mounted on glass slides and subjected to haematoxylineosin staining. Six slides of each sample were randomly chosen. Three investigators who were blinded to the group allocation evaluated the entire coronal pulp on the histological slides.

\section{Results}

\subsection{The morphological assessment of the remineralization}

High-resolution SEM was employed to assess the morphology of the newly precipitated crystals on the demineralized dentinal tissue surface and in dentinal tubules. Fig. 2A shows the dentine surface, on which the orifices of dentinal tubules were distinctly exposed, that we etched with $37 \%$ phosphoric acid for 15 seconds before the remineralization process. Samples in the control group were remineralized with CPP-ACP but without an extrinsic electric field. Only sporadic crystals on the dentine surface were detected after 8 hours of remineralization. The acid-etched dentine was still obvious, and the tubule openings remained patent (Fig. 2B). By contrast, in the experimental groups that had the aid of an extrinsic electric field, abundant crystals precipitated on the dentine surface after merely 3 hours of

Table 1 The micro-hardness values of native, acid-etched and remineralized rabbit dentine

Sample

Mean Knoop hardness value $(\mathrm{KHN}) \pm$ standard deviation (S.D.)

Rabbit native dentine Rabbit acid-etched dentine

Rabbit remineralized dentine in group A for $5 \mathrm{~h}$ remineralization Rabbit remineralized dentine in group $\mathrm{B}$ for $5 \mathrm{~h}$ remineralization

$\begin{aligned} 118.30 & \pm 27.76 \\ 55.06 & \pm 6.87 \\ 126.04 & \pm 6.31 \\ 91.49 & \pm 8.71\end{aligned}$ 

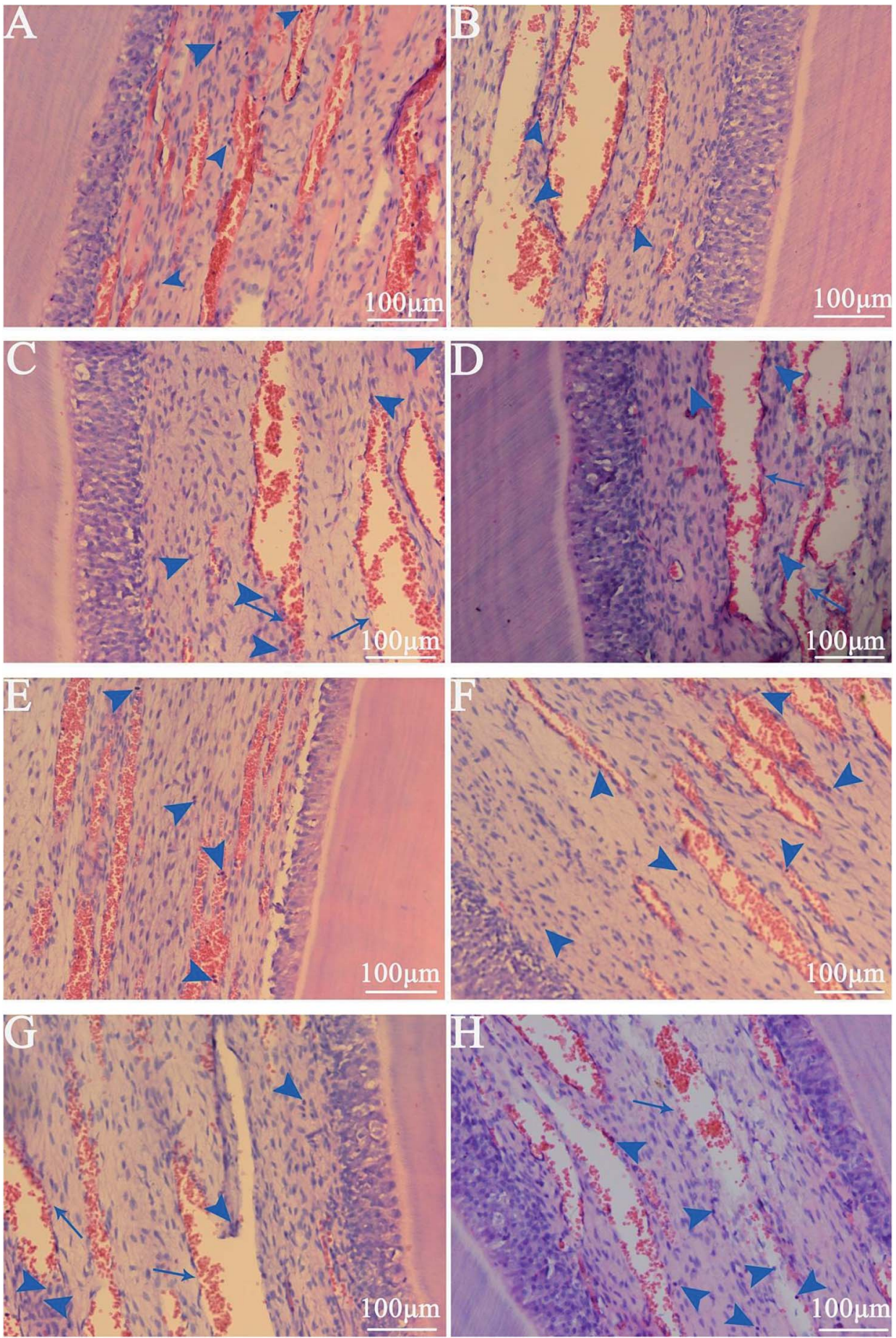

Fig. 6 Histological section photomicrographs. (A) Photomicrograph of pulpal tissue after 3 hours remineralization in group A (the blue arrowhead represents leucocytic infiltration); (B) photomicrograph of pulpal tissue after 5 hours remineralization in group A (the blue arrowhead represents leucocytic infiltration); (C) photomicrograph of pulpal tissue after 8 hours remineralization in group A (the blue arrowhead represents leucocytic infiltration; the blue arrow represents mild hyperemia); (D) photomicrograph of pulpal tissue in control group (the blue arrowhead represents leucocytic infiltration); (E) photomicrograph of pulpal tissue after 3 hours remineralization in group B (the blue arrowhead represents leucocytic infiltration); (F) photomicrograph of pulpal tissue after 5 hours remineralization in group B (the blue arrowhead represents leucocytic infiltration); (G) photomicrograph of pulpal tissue after 8 hours remineralization in group B (the blue arrowhead represents leucocytic infiltration; the blue arrow represents mild hyperemia); $(H)$ photomicrograph of pulpal tissue in control group (the blue arrowhead represents leucocytic infiltration).

remineralization (Fig. 3A and D). At longer durations of remineralization, a significant increase in crystal precipitation and dentinal tubule occlusion was detected. After 8 hours of remineralization, a thick layer of precipitated crystals covered all of the dentine surface. The opening dentinal tubules could not be detected (Fig. 3C and G). 
Furthermore, the strength of the electric currents significantly affected the remineralization processes. In group $\mathrm{A}$, which had a constant current strength of $1.0 \mathrm{~mA}$, precipitated crystals fully covered the intact surface of the acid-etched dentine after 5 hours (Fig. 3B); however, in group B, which had a current strength of $0.5 \mathrm{~mA}$, it took a total of 8 hours to fully cover the acid-etched dentine (Fig. 3G). At the end of the 5 hour period in group A and 8 hour period in group B, crosssectional examination directly and clearly showed that a layer of precipitated crystals had completely covered the dentine surface and that the dentine tubules were occluded (Fig. 4A and B).

\subsection{The characterization of the composition and structure of the precipitated crystals on the remineralized dentine surface}

EDS was applied to measure the $\mathrm{Ca} / \mathrm{P}$ ratio, and $\mathrm{XRD}$ was used to characterize the mineral phase of the precipitated crystals on the remineralized dentine surface in the experimental groups that had the aid of electrophoresis. From the EDS data, it was found that, after 8 hours of remineralization, the $\mathrm{Ca} / \mathrm{P}$ ratios of the new crystals formed in group A and group B were 1.68 and 1.69, respectively (Fig. 3D and $\mathrm{H}$ ), which were similar to the $\mathrm{Ca} / \mathrm{P}$ ratio of the thermodynamically stable hydroxyapatite (1.67). XRD was employed to further determine the mineral phase of the precipitates (Fig. 5). After remineralization, the characteristic diffraction peaks of the remineralized dentine in groups $\mathrm{A}$ and $\mathrm{B}$ of $(002)$ at $2 \theta=25.8^{\circ},(211)$ at $2 \theta=31.9^{\circ},(300)$ at $2 \theta=$ $33.0^{\circ}$ and (004) at $2 \theta=53.2^{\circ}$ were observed, which corresponded to the standard peaks for hydroxyapatite (JCPDS no. 09-0432). At the same time, the peaks of the remineralized dentine were remarkably enhanced compared to those of native dentine, representing a larger quantity of crystallized hydroxyapatite on the acid-etched dentine.

\subsection{The micro-hardness of the remineralized dentine surfaces}

The Knoop hardness values of the rabbits' native, acid-etched and remineralized dentine surfaces that were aided with electrophoresis for 5 hours are shown in Table 1 . The difference in the levels of micro-hardness between the remineralized and acid-etched dentine was significant in group A and group $\mathrm{B}(p<$ 0.01, One-Way ANOVA). The micro-hardness of the remineralized dentine in group A was not significantly different from that of the native dentine ( $p=0.337, t$ test); whereas, significantly different levels of micro-hardness between the native dentine and the remineralized dentine in group B were observed ( $p=$ $0.016, t$ test). The micro-hardness of the remineralized dentine in group A was also significantly different from that of group B $(p=0.004, t$ test $)$.

\subsection{The histological changes of the pulpal tissue after stimulation with an extrinsic electric field}

The haematoxylin-eosin staining showed that, compared with normal pulpal tissue in the control group, the experimental groups showed no vascular collapses, no odontoblast disturbances and no seriously acute inflammatory reactions, such as necrosis or abscess formation (Fig. 6). The walls of the vessels in the pulpal tissue were intact, and all the odontoblasts remained in the palisade pattern. Only slight leucocytic infiltration was detected in all the slides in groups A and B, and also in the control group of healthy pulp tissue. Even after the 8 hour remineralization period, only mild hyperemia was observed (Fig. 6C and G).

\section{Discussion}

In recent decades, plenty of remineralization agents, such as fluoride- and surfactant-based chemicals, have been developed to initiate tooth remineralization. Due to their low efficiency, those remineralization agents are only applied when managing superficial enamel lesions. In view of this, a few strategies have been introduced to accelerate remineralization, for instance, hydrothermal, ${ }^{24}$ laser, ${ }^{25}$ and electrolytic deposition methods. ${ }^{26}$ Nevertheless, most of these methods are environmentally hazardous, or only effective under stringent conditions, like extremely low acidic or high electric field environments, which cannot be translated to clinical applications.

In light of biomineralization, biomimetic mineralization has been explored through mimicking the natural process of mineralization under physiological conditions. A series of biocompatible substances has been employed to promote tooth mineralization so far, such as gelatin,${ }^{27}$ amelogenin,${ }^{28}$ peptide, ${ }^{29}$ polydopamine, ${ }^{30}$ poly(amidoamine) dendrimers ${ }^{31}$ and nacre water-soluble matrix. ${ }^{32}$ Among them, CPP-ACP is a novel remineralizing agent derived from milk. At neutral $\mathrm{pH}$, the 'acidic motif' in CPP is a negatively charged region consisting of three serine phosphate groups followed by two glutamic acid residues, which interact with calcium on the surface of calcium phosphate nanoclusters to form CPP-ACP. ${ }^{33,34}$ CPP-ACP can prevent the decrease of $\mathrm{pH}$ values and provides bioavailable calcium phosphate to treat dental hard tissue lesions by maintaining high concentration gradients of calcium and phosphate ions and ion pairs - especially the neutral ion pair $\mathrm{CaHPO}_{4}^{0}$, which is significantly related to remineralization. ${ }^{35}$ The use of CPP-ACP has been shown not only to prevent a $\mathrm{pH}$ decrease and tooth demineralization, but also to promote remineralization by delivering nanoclusters of calcium and phosphate ions to the tooth surface. Consequently, CPP-ACP has been administered as a colloidal suspension in toothpastes, chewing gums and mouthwashes. There is no doubt regarding its safety. However, the instant therapeutic effects of CPP-ACP for dental hard tissue lesions are insufficient. As revealed in a recent study, even though CPP-ACP forms more stable precipitates than fluoride-based agents, the remineralization rates of CPP-ACP on tooth surfaces are quite low due to its lower diffusion rate. ${ }^{36}$

In this remineralization system, the electrochemical reactions made the water in the vicinity of the cathode hydrolyzed, and this resulted in the $\mathrm{pH}$ value increasing due to hydroxyl aggregation. CPP is negatively charged around neutral $\mathrm{pH} .{ }^{37}$ The alkaline $\mathrm{pH}$ value in the vicinity of the cathode caused an increase of negative charge in CPP. In this study, with the aid of 
an extrinsic electric field and very low current strength, the electrokinetic motions of CPP-ACP (negatively charged) nanocomplexes were initiated. It was found that the extrinsic electric field could accelerate the diffusion of CPP-ACP nanocomplexes from the cathode into the acid-etched dentine. From the SEM observations, it was observed that the extrinsic electric field successfully accelerated the remineralization speed of CPP-ACP. With the aid of electrophoresis, the remineralization speed of CPP-ACP was significantly improved. In comparison with the remineralized dentine in the control group, the amount of precipitated crystals formed in the experimental groups (Fig. 3C and G) after 8 hours remineralization was much more than that in the control group (Fig. 2B). The remineralization speed of CPP-ACP in group A (constant current strength: $1.0 \mathrm{~mA}$ ) was faster than that in group B (constant current strength: $0.5 \mathrm{~mA}$ ). The increased current strength had a powerful effect on the remineralization kinetics of CPP-ACP as many more precipitated crystals were observed in group A than in group B (Fig. 3). Moreover, the micro-hardness of the remineralized dentine was higher in group $\mathrm{A}$ than in group B after 5 hours of remineralization. The electrochemical reactions in the vicinity of the cathode could have led to a hydrolysis reaction that caused hydroxyl aggregation. This eventually resulted in the increase of the local $\mathrm{pH}$ value. The increasing $\mathrm{pH}$ value was beneficial in stimulating the transformation of ACP into mature hydroxyapatite crystals. The mechanism of this novel remineralization system is illustrated in Fig. 7.

To repair dental hard tissue lesions, especially involving dentine, it is essential to recreate an enamel-like hydroxyapatite layer to protect the exposed dentinal tubules from extra stimuli. From our analysis of their chemical compositions and structures, both the EDS and XRD results confirmed that the precipitated crystals that formed on the acid-etched dentine surface in the experimental groups were hydroxyapatite crystals. The transversal micrographs of the remineralized dentine in the experimental groups illustrated that the formed hydroxyapatite layer was tightly fused with the underlying dentine tissue and was arranged in an orderly manner (Fig. $4 \mathrm{~A}$ and B). After the

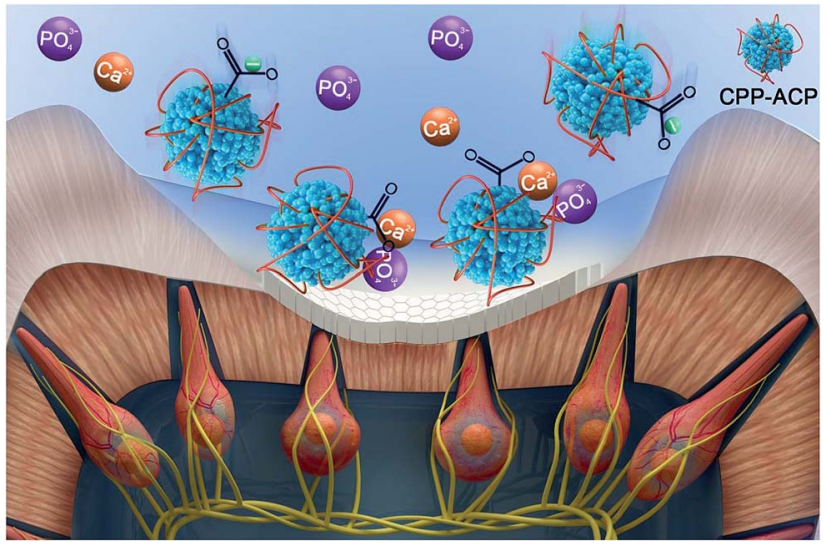

Fig. 7 A schematic image of the electrophoresis-aided CPP-ACP remineralization system. remineralization process, the remineralized dentine was exposed to ultrasound treatment. It was found that the deposited hydroxyapatite layer was not influenced by the ultrasound treatment, which indirectly indicated the existence of a tight binding between the precipitated layer and the dentine substrate. All the above indications imply that the binding between the newly formed hydroxyapatite layer and the underlying dentine tissue was reliable and could effectively resist abrasion. ${ }^{38,39}$ Meanwhile, after 5 hours of remineralization, the microhardness levels of the remineralized dentine in the experimental groups improved significantly. The microhardness of the remineralized dentine in group A, treated with $1.0 \mathrm{~mA}$, recovered to the level of that of the native dentine after 5 hours of remineralization. Based on the above observations, it can be summarized that although the morphology of the new hydroxyapatite layer might not be exactly the same as the native enamel, its composition and structure resemble that of native enamel crystal. Thus, it could effectively protect exposed dentinal tubules from harm.

Electrophoresis/iontophoresis has been widely applied in dermatology, otorhinolaryngology and ophthalmology to improve the therapeutic effects of anti-inflammatory medicine through accurate delivery at the target site. ${ }^{40-42}$ For example, the intensity of the current used in typical iontophoresis devices ranges from $10 \mathrm{~mA}$ to $40 \mathrm{~mA}$ and the intensity has been found to be painless on skin. The applied electric current $(0.5 \mathrm{~mA}$ and 1.0 $\mathrm{mA}$ ) in this study was clearly much smaller than that applied in dermatology, otorhinolaryngology and ophthalmology. Although it was sustained for 5 hours and 8 hours in our study, the haematoxylin-eosin staining results verified that no acute inflammation was observed in the rabbits. In addition, no skin damage related to the prolonged exposure to the anode was detected on the rabbits' head. Thus, the electrophoresis-aided CPP-ACP remineralization system has been proved to be efficient and safe in managing dentine lesions. Meanwhile, considering the feasibility of the electrophoresis-aided CPP-ACP remineralization system in the dental clinic, the 8 hours remineralization process can be completed in several appointments.

One limitation of this study is that the dentine lesion was induced by acidified gel so that the actual clinical situation cannot be revealed. Acidified gel ${ }^{43}{ }^{43}$-cycling ${ }^{44}$ and microbiological incubation ${ }^{45}$ are three common models used for inducing artificial caries. In this study, $37 \%$ phosphoric acid gel was applied to demineralize dentine tissue by exposing dentinal tubules and collagen fibers. Although the acidified gel technique cannot be regarded as a perfect artificial caries model due to lacking saliva and biofilm, it is still widely applied. This is probably because, in comparison with the $\mathrm{pH}$-cycling and microbiological caries models, the degree of demineralization induced by acidified gel is more severe. ${ }^{46}$ The efficiency and effectiveness of the remineralization results obtained from the artificial caries model induced by the acidified gel technique were thus more recognized. Moreover, the lesion induced by acidified gel has been identified as histologically similar to that naturally developed in tooth, especially the lesion in natural root caries. ${ }^{47}$ Another limitation of this study was intrinsically caused by the in vivo design. Although $1.0 \mathrm{~mA}$ and $0.5 \mathrm{~mA}$ were 
chosen for group A and B, respectively, the various sizes of the tooth surface might result in a huge distinction in current density, which is the evaluation indicator for electrochemical reaction. In this in vivo study, rabbits with similar weight and incisor size were carefully chosen in order to minimize the influence of superficial areas on the current density. Considering the difficulties in the control of current density in an in vivo study, in vitro investigations with standardized preparations are needed in future studies.

\section{Conclusions}

Electrophoresis could realize the rapid remineralization and effectively improve the reparative effects of CPP-ACP on demineralized dentine. This study successfully offered a safe and non-invasive route for treating dental hard tissue lesions in dentine. In addition, it might effectively alleviate patients' fear of dental attendance.

\section{Conflicts of interest}

The authors declare no competing interests.

\section{Acknowledgements}

This study was supported by the funding of the National Natural Science Foundation of China (No. 81571018). Y. Y. Zhang, H. M. Wong and Q. L. Li contributed to conception and design of the study and drafted the manuscript. All authors contributed to the acquisition, analysis and interpretation of the data, critically revised the manuscript, gave final approval and agreed to be accountable for all aspects of the work ensuring integrity and accuracy.

\section{References}

1 M. O. Sharif, S. Iram and P. A. Brunton, J. Dent., 2013, 41, 483-492.

2 K. Glockner, Clin. Oral Investig., 2013, 17, 61-62.

3 M. L. Hu, G. Zheng, Y. D. Zhang, X. Yan, X. C. Li and H. Lin, J. Dent., 2018, 75, 12-21.

4 H. M. Wong, C. M. Mak and Y. F. Xu, Noise Health, 2011, 13, 385.

5 G. Humphris and K. King, J. Anxiety Disord., 2011, 25, 232236.

6 X. Xu, L. He, B. Zhu, J. Li and J. Li, Polym. Chem., 2017, 8, 807-823.

7 G. Mortazavi, M. Haghani, N. Rastegarian, S. Zarei and S. Mortazavi, J. Biomed. Phys. Eng., 2016, 6, 41.

8 F. H. van de Sande, P. A. D. R. Rodolpho, G. R. Basso, R. Patias, Q. F. da Rosa, F. F. Demarco, N. J. Opdam and M. S. Cenci, Dent. Mater., 2015, 31, 669-675.

9 N. Beiruti, J. Frencken, M. Van 't Hof and W. van Palenstein Helderman, Community Dent. Oral Epidemiol., 2006, 34, 403409.

10 Y. Y. Zhang, H. M. Wong, C. P. McGrath and Q. L. Li, Sci. Rep., 2018, 8, 8904.
11 F. R. Tay and D. H. Pashley, Biomaterials, 2008, 29, 11271137.

12 Y. Fan, Z. T. Wen, S. Liao, T. Lallier, J. L. Hagan, J. T. Twomley, J.-F. Zhang, Z. Sun and X. Xu, J. Bioact. Compat. Polym., 2012, 27, 585-603.

13 Q. Ruan, Y. Zhang, X. Yang, S. Nutt and J. Moradian-Oldak, Acta Biomater., 2013, 9, 7289-7297.

14 T. Saito, M. Yamauchi and M. A. Crenshaw, J. Bone Miner. Res., 1998, 13, 265-270.

15 J. Ten Cate, J. Dent. Res., 2001, 80, 1407-1411.

16 K. Kawasaki, J. Ruben, I. Stokroos, O. Takagi and J. Arends, Caries Res., 1999, 33, 275-280.

17 T. Y. Ning, X. H. Xu, L. F. Zhu, X. P. Zhu, C. H. Chu, L. K. Liu and Q. L. Li, J. Biomed. Mater. Res., Part B, 2012, 100, 138144.

18 S. Fure, Gerodontology, 2004, 21, 130-140.

19 M. Hayes, F. Burke and P. F. Allen, Root Caries: From Prevalence to Therapy, Karger Publishers, 2017, vol. 26, pp. 1-8.

20 K. Glockner, Clin. Oral Investig., 2013, 17, 61-62.

21 L. W. Li, H. M. Wong, L. Sun, Y. F. Wen and C. P. McGrath, Adv. Nutr., 2015, 6, 828-841.

22 D. Clark and L. Levin, Int. Dent. J., 2016, 66, 249-256.

23 Q. L. Li, T. Y. Ning, Y. Cao, W. B. Zhang, M. L. Mei and C. H. Chu, BMC Biotechnol., 2014, 14, 32.

24 H. Chen, Z. Tang, J. Liu, K. Sun, S. R. Chang, M. C. Peters, J. F. Mansfield, A. Czajka-Jakubowska and B. H. Clarkson, Adv. Mater., 2006, 18, 1846-1851.

25 M. Sun, N. Wu and H. Chen, Sci. Rep., 2017, 7, 9611.

26 W. Ye and X. X. Wang, Mater. Lett., 2007, 61, 4062-4065.

27 S. Busch, Angew. Chem., Int. Ed., 2004, 43, 1428-1431.

28 Y. Fan, J. R. Nelson, J. R. Alvarez, J. Hagan, A. Berrier and X. Xu, Acta Biomater., 2011, 7, 2293-2302.

29 C. Hsu, H. Chung, J. M. Yang, W. Shi and B. Wu, J. Dent. Res., 2011, 90, 88-92.

30 Y. Z. Zhou, Y. Cao, W. Liu, C. H. Chu and Q. L. Li, ACS Appl. Mater. Interfaces, 2012, 4, 6901-6910.

31 J. Li, J. Yang, J. Li, L. Chen, K. Liang, W. Wu, X. Chen and J. Li, Biomaterials, 2013, 34, 6738-6747.

32 X. Li, D. Pan, S. Lin, Z. Zhuang and Z. Lin, CrystEngComm, 2013, 15, 4351-4356.

33 K. J. Cross, N. L. Huq, J. E. Palamara, J. W. Perich and E. C. Reynolds, J. Biol. Chem., 2005, 280, 15362-15369.

34 E. C. Reynolds, Spec. Care Dentist., 1998, 18, 8-16.

35 C. Piekarz, S. Ranjitkar, D. Hunt and J. McIntyre, Aust. Dent. J., 2008, 53, 22-25.

36 I. Pignatelli, A. Kumar, K. Shah, M. Balonis, M. Bauchy, B. Wu and G. Sant, Dent. Mater., 2016, 32, e251-e261.

37 I. Mehdawi, E. A. A. Neel, S. P. Valappil, G. Palmer, V. Salih, J. Pratten, D. A. Spratt and A. M. Young, Acta Biomater., 2009, 5, 2525-2539.

38 Q. Ruan, Y. Zhang, X. Yang, S. Nutt and J. Moradian-Oldak, Acta Biomater., 2013, 9, 7289-7297.

39 J. S. DuPont, Cranio, 2004, 22, 297-303.

40 C. M. Herndon, Pharmacotherapy, 2007, 27, 745-754.

41 A. Semalty, M. Semalty, R. Singh, S. K. Saraf and S. Saraf, Technol. Health Care, 2007, 15, 237-245. 
42 K. J. Cross, N. L. Huq and E. C. Reynolds, Biochemistry, 2016, 55, 4316-4325.

43 L. Silverstone, Br. Dent. J., 1968, 125, 145-157.

44 J. Ten Cate and P. Duijsters, Caries Res., 1982, 16, 201-210. 47 J. M. Mcintyre, J. D. Featherstone and J. Fu, Aust. Dent. J., 45 B. H. Clarkson, J. S. Wefel and I. Miller, J. Dent. Res., 1984, 63, 1186-1189.
46 M. Marquezan, M. E. Sanabe, L. E. R. Filho, J. Hebling, A. C. Guedes-Pinto and F. M. Mendes, Arch. Oral Biol., 2009, 54, 1111-1117. 